\title{
Discriminación de Sexo en una Población Medieval del Sur de la Península Ibérica Mediante el Uso de Variables Simples
}

\author{
Sex Discrimination in a Middle Age Population of the \\ Southern Iberian Peninsula by the Use of Simple Variables
}

Juan Manuel Jiménez-Arenas

JIMÉNEZ-ARENAS, J. M. Discriminación de sexo en una población medieval del Sur de la península Ibérica mediante el uso de variables simples. Int. J. Morphol., 28(3):667-672, 2010.

RESUMEN: Las asignaciones de sexo a los restos osteológicos es fundamental puesto que suponen el primer paso hacia el establecimiento de otros tipos de inferencias biológicas y culturales. Aunque la determinación del sexo es relativamente fácil cuando se recuperan determinadas partes esqueléticas, especialmente la pelvis y el cráneo, resulta más problemática a partir de otros huesos, sobre todo si se trata de fragmentos. En este trabajo se presenta un estudio de discriminación del sexo de los individuos a partir del húmero utilizando variables simples. Los resultados muestran cómo, utilizando la prueba de Lubischew, cada una de las principales partes del húmero aporta, al menos, una variable con porcentajes de discriminación cercanos al 90\%. Estos resultados se comparan con los obtenidos mediante análisis discriminantes. Desde el punto de vista de la aplicación en Arqueología, la prueba de Lubischew se presenta como una herramienta muy útil para la asignación de sexo a individuos de sexo desconocido pertenecientes a muestras suficientemente amplias, siempre y cuando existan asignaciones sexuales previas fiables para un porcentaje alto de los individuos de cada una de aquéllas.

PALABRAS ClAVE: Discriminación del Sexo; Edad Media; Húmero, Prueba de Lubischew; Análisis discriminante.

\section{INTRODUCCIÓN}

Uno de los mayores problemas al que se pueden enfrentar los antropólogos a la hora de trabajar con materiales óseos arqueológicos es la fragmentación del registro, realidad que afecta tanto a los análisis cualitativos como a los cuantitativos. Así, es frecuente recuperar huesos de los que se reconocen sólo partes y/o en los que no se pueden tomar determinadas medidas puesto que los puntos osteométricos están ausentes. Un escenario de elementos óseos completos y bien preservados no es muy habitual, sobre todo cuanto más alejados se encuentren en el tiempo. Es por ello por lo que para alcanzar determinados objetivos es mejor utilizar herramientas metodológicas y técnicas que impliquen el menor número posible de variables. Un caso particular de lo anteriormente expuesto lo tenemos en la discriminación del sexo mediante la utilización de métodos cuantitativos. Los antropólogos forenses que optan por el uso de éstos dedi- can buena parte de sus esfuerzos a buscar formas de aumentar las clasificaciones correctas del sexo de los individuos utilizando el menor número de variables. No obstante, como es bien conocido, no existen patrones universales de discriminación del sexo. Las razones hay que buscarlas en las variabilidades intrapoblacional (Jantz \& Jantz, 1999) e interpoblacional (Lavelle, 1974). Éstas suponen un hándicap importante a la labor de los antropólogos forenses, sobre todo en áreas geográficas y/o tiempos en los que la movilidad, la mixtura y/o los reemplazamientos poblacionales han sido altos. Sin embargo, los inconvenientes derivados de lo anteriormente expuesto se pueden convertir en ventajas, por ejemplo a la hora de evaluar la presencia de elementos alóctonos en las muestras.

El húmero ha comenzado a recibir una mayor atención a la hora de discriminar (estadísticamente) el sexo

Departamento de Prehistoria y Arqueología. Campus Universitario de Cartuja, s/n. 18071 Granada, España.

Este trabajo se ha podido llevar a cabo gracias a un Contrato de Reincorporación de Doctores del Plan Propio de la Universidad de Granada y al apoyo del grupo de investigación HUM-607 (Junta de Andalucía, España) y de los proyectos de investigación CGL2008-04896 y HAR2008-04577, Ministerio de Ciencia e Innovación, España. 
de los individuos desde hace relativamente poco tiempo, en la década de los ochenta. Una posible razón es que, como se ha apuntado frecuentemente, no todos los huesos reflejan las diferencias sexuales de la misma manera, ni con igual intensidad, y los esfuerzos se han centrado en otras partes esqueléticas que, dejando al margen la pelvis, reflejan mejor el dimorfismo sexual: por ejemplo, el fémur. No obstante, el creciente aumento del interés por el húmero, a lo largo de las tres últimas décadas, ha puesto de manifiesto que también se trata de un elemento óseo interesante para vislumbrar las diferencias entre los sexos. La procedencia de las muestras es variada: para el continente asiático se cuenta con estudios para poblaciones de China, Tailandia y Japón (Iscan et al., 1998) e India (Singh \& Singh, 1972), para África con dos estudios en Sudáfrica (Steyn \& Iscan, 1999; Robinson \& Bidmos, 2009), para el americano con poblaciones de Estados Unidos (Dittrick \& Suchey, 1986; Holman \& Bennet, 1991) y Guatemala (Ríos Frutos, 2005) y, finalmente, para Europa con colecciones procedentes de Portugal (Carretero et al., 1995), Alemania (Mall et al., 2001), España (Hernández et al., 1991; Alemán et al., 1997) y Grecia (Kranioti \& Michalodimitrakis, 2009).

El objetivo del presente trabajo es discriminar el sexo de los individuos a partir de los húmeros de la muestra procedente de la necrópolis musulmana de La Torrecilla (Arenas del Rey, Granada, España), apelando a un criterio de máxima parsimonia, esto es, utilizar, variables lineales simples una a una, y contrastando los resultados obtenidos mediante dos técnicas estadísticas diferentes: el análisis discriminante y la prueba de Lubischew. Los resultados son comparados con los de otras poblaciones.

\section{MATERIAL Y MÉTODO}

Para este trabajo se han medido un total de 73 húmeros (33 correspondientes a mujeres y 40 a hombres). Las medidas que se han utilizado son estándares y reflejan las tres principales partes en las que se divide dicho hueso, epífisis proximal ( 2 variables), diáfisis ( 2 variables) y epífisis distal (2 variables), así como la longitud del mismo (2 variables) (Tabla I).

Para evaluar la discriminación entre los individuos de sexo conocido se ha utilizado la prueba de Lubischew. Ésta, que se basa en la prueba t de Student, permite evaluar el grado de solapamiento entre las distribuciones muestrales de dos poblaciones y, con ello, determinar qué capacidad tiene dicha variable de discriminar entre ambas (Lubischew, 1962). Y, lo que es más importante, lo hace no sólo en función de la muestra utilizada sino que, como en cualquier prueba en la que se evalúa una distribución de probabilidad, los resultados son extrapolables a la población en su conjunto. El coeficiente de discriminación (K) se calcula siguiendo la fórmula $\mathrm{K}=\left(\overline{\mathrm{X}}_{\mathrm{a}}-\overline{\mathrm{X}}_{\mathrm{b}}\right) / 2 \mathrm{Sx} \mathrm{X}^{2}$, donde $\overline{\mathrm{X}}_{\mathrm{a}} \mathrm{y} \overline{\mathrm{X}}_{\mathrm{b}}$ son las medias de la variable $\mathrm{X}$ en las dos muestras comparadas (A y B) y $\mathrm{Sx}^{2}$ es la varianza mancomunada para ambas muestras. Dado que el porcentaje de clasificaciones erróneas a partir de la variable $\mathrm{X}$ (es decir, el solapamiento entre las muestras) es aproximadamente la probabilidad de que una desviada normal exceda el valor $\sqrt{ } \mathrm{K} / 2$, conforme mayor sea $\mathrm{K}$ mejor será el carácter $\mathrm{X}$ a la hora de discriminar. Como en cualquier prueba de la estadística paramétrica, la de Lubischew requiere que las distribuciones de los valores de la variable en cuestión en las muestras comparadas sean aproximadamente normales y de

Tabla I. Variables utilizadas en el presente estudio.

\begin{tabular}{|c|c|c|c|}
\hline Variable & Abreviatura & Descripción & Instrumento de medida \\
\hline Longitud máxima & HLmax & $\begin{array}{l}\text { Distancia entre el punto más proximal de la cabeza del } \\
\text { húmero y el punto más distal de la tróclea del húmero }\end{array}$ & Plancha osteométrica \\
\hline Longitud fisiológica & HLfis & $\begin{array}{l}\text { Distancia entre el punto más alto de la cabeza del húmero al } \\
\text { punto más distal del capítulo }\end{array}$ & Plancha osteométrica \\
\hline $\begin{array}{l}\text { Diámetro vertical de } \\
\text { la cabeza }\end{array}$ & HHDsi & $\begin{array}{c}\text { Distancia entre los márgenes proximal y distal de la cara } \\
\text { articular humeral }\end{array}$ & Pie de rey \\
\hline $\begin{array}{l}\text { Diámetro transversal } \\
\text { de la cabeza }\end{array}$ & HHDap & $\begin{array}{l}\text { Distancia máxima de la superficie articular, en sentido } \\
\text { anteroposterior, tomada sobre el cuello anatómico }\end{array}$ & Pie de rey \\
\hline $\begin{array}{l}\text { Diámetro máximo en } \\
\text { la mitad }\end{array}$ & HDDmax & $\begin{array}{c}\text { En mitad de la diáfisis, se rota el hueso hasta obtener la } \\
\text { distancia máxima }\end{array}$ & Pie de rey \\
\hline $\begin{array}{l}\text { Diámetro mínimo en } \\
\text { la mitad }\end{array}$ & HDDmin & Medida tomada en perpendicular a la anterior & Pie de rey \\
\hline Anchura epicondilar & HEW & $\begin{array}{c}\text { Distancia entre los puntos más medial y lateral de los } \\
\text { epicóndilos }\end{array}$ & Pie de rey \\
\hline $\begin{array}{l}\text { Anchura de la } \\
\text { superficie articular }\end{array}$ & HASW & $\begin{array}{c}\text { Distancia entre el margen lateral del capítulo del húmero y el } \\
\text { límite medial de la superficie articular }\end{array}$ & Pie de rey \\
\hline
\end{tabular}


varianza similar. Por tanto, antes de llevar a cabo la prueba de Lubischew es necesario evaluar dos hipótesis nulas: la primera, que las distribuciones de las muestras en cada una de las variables sean normales y la segunda, que las varianzas de las muestras que se comparan para cada una de las variables no sean significativamente diferentes entre sí (homocestaticidad). Para ello se han empleado las pruebas de Shapiro-Wilk (normalidad de las distribuciones de las muestras) y de Levene (homocestaticidad u homogeneidad de las varianzas). Por su parte, el análisis discriminante se ha convertido en una de las principales herramientas estadísticas para generar modelos predictivos en los que se pronostica el grupo de pertenencia de cada una de las observaciones. Se generan funciones discriminantes basadas, en este caso, en una única variable que provee la mejor discriminación posible entre los grupos. Para evaluar la bondad de los resultados de los análisis discriminantes se ha utilizado el método de la validación cruzada. Todas las pruebas estadísticas, salvo la de Lubischew, se han realizado con el software PASW 18.0 .

\section{RESULTADOS}

Los estadísticos descriptivos correspondientes a las distintas variables están resumidos en la Tabla II. Las pruebas de Shapiro-Wilk para evaluar si se puede rechazar la hipótesis nula (la muestra proviene de una distribución nor- mal) arrojan los siguientes resultados: todas las variables testadas presentan distribuciones que no pueden ser rechazadas como normales, con las excepciones de las anchuras epicondilares de las mujeres y de los hombres (Tabla II). Respecto a la prueba de Levene para evaluar la homocestaticidad se puede rechazar la hipótesis nula (las varianzas de las muestras de mujeres y hombres son homogéneas para una misma variable) para la longitud máxima del húmero y para los diámetros de la cabeza el mismo (Tabla III). De cualquier manera, los valores por los cuales se rechazan las hipótesis nulas de normalidad y homocestaticidad no son muy elevados y por tanto se les puede aplicar la prueba de Lubischew.

El porcentaje de discriminación (Tabla II) alcanzado por el conjunto de las variables humerales usando la prueba de Lubischew oscila entre el $85,1 \%$ para la longitud fisiológica y el $91 \%$ para el diámetro superoinferior de la cabeza. Entre ambas medidas se situarían la anchura biepicondilar $(85,3 \%)$, los diámetros máximo $(86,4 \%)$ y mínimo de la diáfisis $(87,7 \%)$, la longitud máxima $(88,1 \%)$, la anchura de la superficie articular $(88,5 \%)$ y el diámetro anteroposterior de la cabeza $(89,07 \%)$. Por su parte, los análisis discriminantes (mediante validación cruzada) para variables simples proporcionan resultados bastante similares a los anteriores (Tabla II). Empero, existen ciertas diferencias en cuanto al poder discriminador de cada una de las variables. Así, la que proporciona un mayor porcentaje de reclasificaciones correctas es la longitud fisiológica del húmero $(90,8 \%)$ y la que menos la anchura mínima en la mitad de la diáfisis del mismo (83,3\%).

Tabla II. Estadísticos descriptivos, valor P del estadístico de Shapiro-Wilk y prueba de Lubischew. Abreviaturas de las variables, ver Tabla I. (h) hombres; (m) mujeres; N: número de observaciones; X promedio; SD: desviación estándar; K: coeficiente de discriminación; $\mathrm{R}: \sqrt{\mathrm{K}} / 2$. Si el valor P calculado a partir del estadístico de Shapiro-Wilk es $<0,05$ se rechaza la hipótesis nula, esto es, que las distribuciones de las muestras son normales.

\begin{tabular}{lcccccccc}
\hline La Torrecilla (h) & HLmax & HLfis & HHDap & HHDsi & HDDmax & HDDmin & HEW & HASW \\
\hline $\mathrm{N}$ & 39 & 37 & 17 & 35 & 37 & 37 & 40 & 34 \\
$\bar{X}$ & 320,20 & 315,90 & 41,34 & 45,15 & 22,28 & 17,58 & 61,56 & 45,09 \\
$\mathrm{SD}$ & 15,99 & 21,15 & 2,32 & 3,04 & 1,61 & 1,32 & 3,62 & 3,09 \\
$\mathrm{P}$ (Shapiro-Wilk) & 0,51 & 0,29 & 0,11 & 0,3 & 0,63 & 0,13 & $<0,05$ & 0,08 \\
\hline La Torrecilla (m) & HLmax & HLfis & HHDap & HHDsi & HDDmax & HDDmin & HEW & HASW \\
\hline $\mathrm{N}$ & 31 & 28 & 11 & 20 & 29 & 29 & 33 & 25 \\
$\overline{\mathbf{X}}$ & 284,33 & 279,33 & 36,16 & 37,98 & 19,12 & 14,85 & 53,70 & 38,41 \\
$\mathrm{SD}$ & 12,48 & 11,20 & 1,42 & 1,88 & 1,18 & 0,96 & 3,90 & 2,28 \\
$\mathrm{P}$ (Shapiro-Wilk) & 0,10 & 0,16 & 0,37 & 0,40 & 0,86 & 0,22 & $<0,02$ & 0,23 \\
\hline La Torrecilla (h)/La Torrecilla (m) & HLmax & HLfis & HHDap & HHDsi & HDDmax & HDDmin & HEW & HASW \\
\hline K & 3,04 & 2,17 & 3,29 & 3,57 & 2,42 & 2,69 & 2,20 & 2,89 \\
R & 1,23 & 1,04 & 1,28 & 1,34 & 1,10 & 1,16 & 1,05 & 1,20 \\
\% solapamiento & 11,93 & 14,92 & 10,03 & 9,01 & 13,57 & 12,30 & 14,69 & 11,51 \\
\% discriminación & 88,1 & 85,1 & 89,1 & 91,0 & 86,4 & 87,7 & 85,3 & 88,5 \\
\% reclasificaciones correctas & 87,1 & 90,8 & 89,3 & 89,1 & 87,9 & 83,3 & 87,7 & 86,4 \\
\hline
\end{tabular}


Tabla III. Prueba de Levene para evaluar la homogeneidad de las varianzas de los grupos establecidos en cada variable. Si el valor P calculado a partir del estadístico de Levene es $<0,05$ se rechaza la hipótesis nula, esto es, que las varianzas de los distintos grupos sean homogéneas.

\begin{tabular}{ccccccccc}
\hline & HLmax & HLfis & HHDap & HHDsi & HDDmax & HDDmin & HEW & HASW \\
\cline { 2 - 8 } $\mathrm{P}($ Levene $)$ & $<0,05$ & 0,05 & $<0,01$ & $<0,02$ & 0,16 & 0,09 & 0,84 & 0,07 \\
\hline
\end{tabular}

Tabla IV. Diámetro superoinferior (vertical) de la cabeza del húmero. Comparación de los resultados obtenidos en este estudio con los publicados en un grupo seleccionado de trabajos.

\begin{tabular}{|c|c|c|c|}
\hline Población & Variable & $\%$ & Fuente \\
\hline Surafricanos negros & Diámetro vertical de la cabeza & $\sim 91,0$ & Steyn \& Iscan, 1999 \\
\hline Surafricanos negros & Diámetro vertical de la cabeza & $\sim 84,0$ & Steyn \& Iscan, 1999 \\
\hline Chinos & Diámetro vertical de la cabeza & $\sim 80,5$ & Iscan et al., 1998 \\
\hline Japoneses & Diámetro vertical de la cabeza & 87,3 & Iscan et al., 1998 \\
\hline Tailandeses & Diámetro vertical de la cabeza & 90,4 & Iscan et al., 1998 \\
\hline Alemanes & Diámetro vertical de la cabeza & 90,4 & Mall et al., 2001 \\
\hline $\begin{array}{l}\text { Norte americanos, Central California } \\
\text { (Early Horizon) }\end{array}$ & Diámetro vertical de la cabeza & 85,7 & Dittrick y Suchey, 1986 \\
\hline $\begin{array}{l}\text { Norte americanos, Central California } \\
\text { (Middle and Late Horizon) }\end{array}$ & Diámetro vertical de la cabeza & 90,3 & Dittrick \& Suchey, 1986 \\
\hline Portugueses & Diámetro vertical de la cabeza & 89,0 & Carretero et al., 1995 \\
\hline Españoles & Diámetro vertical de la cabeza & 91,3 & Alemán et al., 1997 \\
\hline Españoles & Diámetro vertical de la cabeza & 91,0 & Este estudio \\
\hline Guatemaltecos & Diámetro máximo de la cabeza & 95,5 & Ríos Frutos, 2005 \\
\hline Cretenses & Diámetro vertical de la cabeza & 89,9 & Kranioti \& Michalodimitrakis, 2009 \\
\hline
\end{tabular}

\section{DISCUSIÓN}

El valor del húmero como elemento osteológico discriminador del sexo de los individuos ha sido puesto de manifiesto tanto en poblaciones contemporáneas como en pretéritas. En el caso de la muestra de La Torrecilla también es así, presentando un alto porcentaje de discriminación $>85 \%$ para todas las variables, usando la prueba de Lubischew, e igualmente superior a dicho porcentaje, salvo para una variable (HDDmin), cuando se recurre al análisis discriminante mediante validación cruzada. Estos resultados son superiores al $~ 80 \%$ de discriminación que presentaron Hernández et al. utilizando un análisis discriminante (stepwise) con dos variables humerales (Hernández et al.) y similares a los obtenidos por el autor de este trabajo para variables simples femorales de esta misma población (Jiménez-Arenas, 2010). Los resultados de la aplicación de la prueba de Lubischew en el presente estudio son, asimismo, coherentes con los obtenidos por diferentes autores que han empleado poblaciones distribuidas a lo largo de África, América, Asia y Europa. Según France, las epífisis distales diferenciarían mejor el sexo de los individuos que las restantes partes del húmero (epífisis proximal y diáfisis) puesto que reflejaría mejor que éstas las cargas derivadas de las funciones de los brazos (France, 1983). Sin embargo, los estudios de diferentes poblaciones en el mundo ponen de manifiesto que existe una gran disparidad entre las partes del húmero que resultan más fiables para la discriminación de sexo. Así las cosas, del conjunto de las variables simples utilizadas, las que mejor discriminan el sexo de los individuos de la necrópolis de La Torrecilla a partir de la prueba de Lubischew son las correspondientes a la cabeza del húmero, coincidiendo con lo predicho por France y con los resultados de otros estudios precedentes (Mall et al.; Dittrick \& Suchey; Steyn \& Iscan; Alemán et al.; Ríos Frutos). No obstante, también se puede deber al uso de diferentes técnicas discriminantes puesto que, cuando se utiliza el análisis discriminante mediante validación cruzada, la variable que mejor discrimina es una longitud (HLfis). El hecho de la inexistencia de un patrón de variabilidad común a todas las 
poblaciones, y por ende de discriminación del sexo, se podría relacionar también con las variabilidades intrapoblacional (Jantz \& Jantz) e interpoblacional (Lavelle). Entre los factores que implican variabilidad intrapoblacional cabe destacar el tiempo, el cual ha afectado a la morfometría de los huesos en diferente grado (Jantz \& Jantz). Teniendo en cuenta que la necrópolis de La Torrecilla se amortiza tras al menos cuatro siglos de uso (Du Souich, 1979) y que la discriminación es alta para la mayoría de las variables, podría plantearse que dicha población fuese bastante homogénea y que cupiese la posibilidad de que no estuviera sujeta a reemplazos poblacionales vinculados a las invasiones almorávide y almohade y/o a cambios seculares producidos por variaciones sustanciales en las formas de vida. Desde el punto de vista del porcentaje de discriminación, la parte del presente estudio dedicada a la prueba de Lubischew también arroja resultados parecidos a algunos de los publicados anteriormente (Tabla IV). Más concretamente, el diámetro vertical de la cabeza del húmero, que es la variable para la que el porcentaje de discriminación entre las muestras de mujeres y de hombres es mayor, presenta un valor muy similar al de poblaciones tan dispares como la norteamericana-California Central, Middle and Late Horizon- (Dittrick \& Suchey), la portuguesa (Carretero et al.), la española contemporánea de Granada (Alemán et al.), la de surafricanos negros (Steyn \& Iscan), la tailandesa (Iscan et al.) y la alemana (Mall et al.). Para la población guatemalteca la discriminación es sensiblemente mayor (Ríos Frutos) y menor si se compara con la de surafricanos blancos (Steyn \& Iscan), la china y la japonesa (Iscan et al.) y la norteamericana -Central California, Early Horizon- (Dittrick \& Suchey). Por tanto, puede considerarse que el escaso solapamiento en las variables simples y el elevado grado de discriminación para éstas en la muestra de La Torrecilla denotan que el dimorfismo sexual en el húmero es alto, tal y como fue puesto de manifiesto en un estudio previo (Hernández et al.).

Una de las ventajas del uso de variables simples para discriminar el sexo de los individuos es que presenta una gran aplicabilidad en Antropología Forense y en Arqueología, donde los esqueletos pueden aparecer fragmentados y/o fuertemente alterados y en los que no siempre es posible tomar un conjunto amplio de medidas. Así, cada una de las partes del húmero ofrece, de manera independiente, niveles de discriminación próximos al $90 \%$. Por tanto, resulta posible identificar con un grado de fiabilidad bastante alto el sexo a partir de cada una de las epífisis y de la diáfisis.

Por otro lado, frente a los análisis discriminantes con una sola variable, cuyos puntos de corte pueden estar forzados por la presencia de valores extremos, la prueba de Lubischew permite una evaluación de la discriminación/ solapamiento de la muestra y de la población, con lo cual, en principio, es menos vulnerable a las contingencias de la muestra estudiada. De esta manera, la seguridad para asignar los sexos a los individuos inciertos de la misma población será mucho mayor que cuando se emplea el análisis discriminante con una sola variable. Además, puesto que la prueba de Lubischew es independiente del tamaño muestral, está especialmente indicada cuando el número de individuos es reducido. Esto la convierte en un test estadístico especialmente indicado para contextos osteoarqueológicos en los cuales las muestras se presentan limitadas en su cantidad. Por el contrario, no permite el cálculo de algoritmos similares al de las funciones discriminantes que posibilite la aplicación directa en otras poblaciones. Por todo ello, y debido a las diferencias en los resultados obtenidos según el test estadístico empleado, se recomienda utilizar conjuntamente el análisis discriminante y la prueba de Lubischew, puesto que los porcentajes de discriminación del sexo de los individuos, un objetivo fundamental de la Antropología Forense, se ven sensiblemente mejorados.

AGRADECIMIENTOS. Al personal del Laboratorio de Antropología Física de la Universidad de Granada, especialmente a los profesores Philippe du Souich y Miguel Botella por haber permitido al autor de este trabajo utilizar los materiales de la colección de La Torrecilla custodiada en dicha institución. A María Ruiz por sus correcciones de estilo y a Paul Palmqvist por sus comentarios y discusiones que, sin duda, han mejorado sustancialmente las versiones preliminares de este manuscrito.

JIMÉNEZ-ARENAS, J. M. Sex discrimination in a middle age population of the Southern Iberian peninsula by the use of simple variables. Int. J. Morphol., 28(3):667-672, 2010.

SUMMARY: Sex determination on osteological remains is key since it is the initial step to establish another kind of biological and cultural inferences. Though sex determination arises easily when some particular parts of the skeleton are collected, specifically the pelvis and the skull, this target is more difficult from other bones, specifically when they are fragmented. In the present paper a study on sex discrimination based on simple humeral variables is shown. The use of the Lubischew's test show that at least one variable in each part of the humerus provides a discriminant percentage close to $90 \%$. These results are compared with those obtained by discriminanting analysis. From an archaeological point of view, the Lubischew's test is a useful tool to infer the sex of the uncertain individuals belonging to large enough samples with previous reliable sex assigments.

KEY WORDS: Sex discrimination; Middle Age; Humerus; Lubischew's Test; Discriminant Analysis. 


\section{REFERENCIAS BIBLIOGRÁFICAS}

Alemán, I.; Botella, M. C. \& Ruíz, L. Determinación del sexo en el esqueleto postcraneal. Estudio de una población mediterránea actual. Archiv. Esp. Morfol., 2:69-79, 1997.

Carretero, J. M.; Lorenzo, C. \& Arsuaga, J. L. Análisis multivariante del húmero en la colección de restos identificados de la Universidad de Coimbra. Antropol. Port., 13:139-56, 1995.

Dittrick, J. \& Suchey, J. M. Sex determination of prehistoric central California skeletal remains using discriminant analysis of the femur and humerus. Am. J. Phys. Anthropol., 70(1):3-9, 1986.

Du Souich, Ph. Estudio antropológico de la necrópolis medieval de Torrecilla (Arenas del Rey, Granada). Antrop. y Paleoecol. Humana, 1:27-40, 1979.

France, D. L. Sexual dimorphism in the human humerus. Boulder, University of Colorado, 1983.

Hernández, M.; Pérez-Pérez, A. \& Jiménez, S. Dimorfismo sexual y asimetrías de húmero y fémur en poblaciones de la Península Ibérica. En: Botella, M.; Jiménez, S.; Ruiz, L. \& Du Souich, P. (Eds.). Nuevas perspectivas en Antropología. Granada, Universidad de Granada, 1991.

Holman, D. J. \& Bennett, K. A. Determination of sex from arm bone measurements. Am. J. Phys. Anthropol., 84:421-6, 1991.

Iscan, M. Y.; Loth, S. R.; King, C. A.; Shihai, D. \& Yoshino, M. Sexual dimorphism in the humerus: A comparative analysis of Chinese, Japanese and Thais. Forensic Sci. Int., 98:17-29, 1998.

Jantz, L. M. \& Jantz, R. L.Secular change in long bone length and proportion in the United States, 1800-1970. Am. J. Phys. Anthropol., 110:57-67, 1999.

Jiménez-Arenas, J. M. Discriminación del sexo en una población medieval del sur de la Península Ibérica. Cuadernos de Prehistoria y Arqueología de la Universidad de Granada, 2010. En prensa.

Kranioti, E. F. \& Michalodimitrakis, M. Sexual dimorphism of the humerus in contemporary Cretans--a populationspecific study and a review of the literature. J. Forensic. Sci., 54(5):996-1000, 2009.
Lavelle, C. L. An analysis of the human femur. Am. J. Anat., 141(3):415-26, 1974.

Lubischew, A. A. On the use of discriminant functions in taxonomy. Biometrics 18:455-77, 1962.

Mall, G.; Hubig, M.; Büttner, A.; Kuznik, J.; Penning, R. \& Graw, M. Sex determination and estimation of stature from the long bones of the arm. Forensic Sci. Int., 117:2330, 2001.

Ríos Frutos, L. Metric determination of sex from the humerus in a Guatemalan forensic sample. Forensic Sci. Int., 147:153-7, 2005.

Robinson, M. S. \& Bidmos, M. A. The skull and humerus in the determination of sex: Reliability of discriminant function equations. Forensic Sci. Int., 186:86.e1-5, 2009.

Singh, S. \& Singh, S. P. Identification of sex from the humerus. Indian J. Med. Res., 60:1061-6, 1972.

Steyn, M. \& Iscan, M. Y.Osteometric variation in the humerus: sexual dimorphism in South Africans. Forensic Sci. Int., 106:77-85, 1999.

Dirección para correspondencia:

Juan Manuel Jiménez-Arenas

Departamento de Prehistoria y Arqueología.

Campus Universitario de Cartuja, s/n. 18071

Granada

ESPAÑA

Email: jumajia@ugr.es.

Recibido : 03-02-2010

Aceptado: 07-06-2010 